\title{
A Novel Human-Machine Interface for Guiding: The NeoASAS Smart Walker
}

\author{
Maria M. Martins ${ }^{\# 1}$, Anselmo Frizera-Neto ${ }^{* 2}$, Eloy Urendes ${ }^{\S 3}$, Cristina P. Santos ${ }^{\sharp 4}$, Ramón Ceres ${ }^{\S 5}$ \\ \# Industrial Electronics Department, University of Minho, Guimarães, Portugal \\ ${ }^{1}$ mmartins@dei.uminho.pt \\ ${ }^{4}$ cristina@dei.uminho.pt \\ "Electrical Engineering Department, Federal University of Espirito Santo (UFES) \\ Av. Fernando Ferrari, 514. Vitoria-ES, Brazil \\ 2 anselmodele.ufes.br \\ ${ }^{\S}$ Bioengineering Group, Spanish National Research Council (CSIC) \\ Crta. Campo Real Km 0.200-Arganda del Rey. Madrid \\ ${ }^{3}$ eloy .urendes @car.upm-csic.es \\ ${ }^{5}$ ceres@iai.cic.es
}

\begin{abstract}
In an aging society it is extremely important to develop devices, which can support and aid the elderly in their daily life. This demands tools that extend independent living and promote improved health. In this work it is proposed a new interface approach integrated into a walker. This interface is based on a joystick and it is intended to extract the user's movement intentions. The interface is designed to be userfriendly, simple and intuitive, efficient and economic, meeting usability aspects and focused on a commercial implementation, but not being demanding at the user cognitive level. Preliminary sets of experiments were performed which showed the sensibility of the joystick to extract navigation commands from the user. These signals presented a higher frequency component that was attenuated by a Benedict-Bordner $\mathrm{g}$-h filter. The presented methodology offers an effective cancelation of the undesired components from joystick data, allowing the system to extract in real-time voluntary user's navigation commands. Based on this real-time identification of voluntary user's commands, an approach to the control architecture of the robotic walker is being developed, in order to obtain stable and safe user assisted locomotion.
\end{abstract}

\section{INTRODUCTION}

Mobility is a fundamental requirement for our daily life. If mobility degrades by any reason our quality of life will decline severely. The number of people with reduced mobility capabilities increases from year to year [1]. Loosing complete or part of mobility, affects not only the ability to walk but also the ability to perform personal tasks, which is a major determinant in life quality and causes dependence of others in daily life.

Thus, it becomes more and more relevant to find ways and tools to compensate, improve or help to restore and enhance this people mobility. These will extend independent living and promote improved health. So, recent advances in robot technology have been developed for the elderly and lower limb disable people. There are examples that include orthoses, prostheses, canes and walkers. These devices provide users with residual capacity of walking.
In particular walkers present great rehabilitation and functional compensation potentials [2] and thus in recent years, their development has been growing. These potentials arise due to the use of the user's residual capacities, trying to maintain and enhance them through functional compensation. Thus, they produce an opposite effect comparatively to the wheelchairs, that have an incapacitate effect on users due to the decrease in the use of the lower limbs.

There are several types of walkers that can be distinguished in many ways, such as, technological complexity, size and structure, among others. However, it is possible to highlight two major groups of walkers: conventional and smart walkers.

Conventional walkers [2] are passive, and they offer a stable platform that increases user's anterior and lateral support, adding an important user's partial body weight support. By being passive, they have a simple structure and are low cost. However, they can be difficult to manipulate because of their size, when climbing stairs or passing through doors. Additionally, they can be unsafe to use in uneven/slope terrain. Furthermore, these walkers reduce the movements of the arms during gait, cause bad posture, and especially, cause an unnatural gait due to how they need to be pushed and/or lifted during gait.

Smart walkers have emerged usually with the same architecture as the conventional ones but they include additional robotic and electronic components, that promote a better assistance to gait, especially considering navigation [3], gait monitoring [4], and partial body weight support [4][5]. However, some of these devices are still too complex to use. These devices have to be built considering that many users have not only problems to walk, but also have cognitive and sensor impairments. For instance, elderly people present usually slower behaviour and are not familiar with mechatronic devices. Furthermore, they need to continuously use their own device in daily routines, such as, go to the bathroom, elevator, etc. In order to overcome these cognitive and sensory deficiencies, studies regarding the development of 
interfaces that establish a direct control of the user are increasing in the literature [3][5][6][9-12]. These interfaces have to be user-friendly, natural and transparent to the users, not being demanding at their cognitive level. The idea is these interfaces have to be able to read and interpret the user's intentions and command the device accordingly.

In this work, it is presented a new interface approach developed in CSIC, and integrated on the NeoASAS walker. Such interface is designed to be simple, intuitive and economic, meeting usability aspects and focused on a commercial implementation. The interface integrates a joystick into centre of the walker upper base support. A preliminary set of experiments were performed with healthy volunteers and no motorization in the device. An analysis of this interface data, enabled to identify two main components. One is a high-frequency component caused by the vibrations introduced by the ground and wheels irregularities. This component is attenuated through a Benedict-Border $\mathrm{g}$-h filter [6]. The other component is related to the user's navigation commands, which are going to be used in the guidance of the walker and their inference is fundamental for an efficient control of the device during assistive gait.

In Section II a critical review regarding the interfaces that are being developed for Smart Walkers is presented. Section III introduces the NeoAsas walker and its background. Section IV describes the NeoASAS interface and discusses the acquisition of the signal components related to the user's navigation commands. Section $\mathrm{V}$ introduce the filter strategy chose to track the acquired signals. The performance of the filter strategy when tracking the user commands in real data is discussed in Section VI. Finally, conclusions and future work are discussed in Section VII.

\section{USER-MACHINE INTERFACES IN SMART WALKERS}

Systems or machines have increasingly evolved in nature and capacity, so now they can assist humans in tasks of higher level of abstraction. The elements that establish a bridge of interaction between human and machine, called interfaces, must establish a dialogue as natural and efficient as possible [7]. In interfaces, the role of the sensors is very important since they measure the environment and human interaction. Additionally, there are actuators that transmit information to the individual cognitive robotics in order to complement their sensory information about the task [8]. The interface should be able to adapt to users with different levels of physical and cognitive capacities and this adaption should be done in a user-friendly manner.

Interfaces can be classified as direct or indirect, depending on whether the user commands or intentions pass directly to the device.

Direct interfaces require a manual operation from the patient. One example of a walker that has this type of interface is ASAS (Andador Pseudorobótico de Alta Seguridad) that was designed by the Bioengineering Group at CSIC [9]. Its interface is based on push buttons installed on each handle such that each motor is controlled by the corresponding push- button. In order to move straight, the user has to press both buttons and to turn has to press only one of them.

Joysticks provide for another example of direct interfaces. Hashimoto et al. [10] developed a walker that is driven with a joystick. This joystick besides transmitting to the motors the user's intention of direction, it has a user-friendly hand force feedback system that allows the user to perceive obstacles and thus walk with greater safety. According to a repulsive force from the joystick, the user can know the distance and direction to the obstacles.

Force sensors have been the focus of direct interfaces, because by detecting the user's intentions though physical interaction, the force sensor interface offers a naturally and intuitively way to reach that goal.

Morris et al. [3] developed a robotic walker based on the XR4000 platform with two-force sensors embedded into the handlebars. By incorporating force sensors inside the handlebars, the user can maintain a steady hold and manipulate the robotic walker in a manner more consistent with contemporary roller-based walkers. Each handlebar is equipped with a prismatic handgrip that is motion constrained. Semi-pliable foam is inserted between the handgrip and motion stops to dampen the displacement exhibited by the grippers. A pair of force-sensing resistors is embedded into the foam to detect pressure when force is exerted along the handlebar. These pressure readings are transformed into planar translational and rotational velocities.

The Personal Adaptive Mobility Aid (PAM-AID) [11] is a walker that doesn't have motorized locomotion. The electronic system only controls the orientation of the front wheel, based on the guidance information acquired by an intuitive interface that is similar to the handlebar of a bicycle that can rotate $+/-15^{\circ}$. The handlebar is spring loaded and when no torque is applied it will return to its zero position.

The Guido [12] (originally known as the PAM-AID) is a robotic walker that is directed by the user with spring-loaded handlebars that are equipped with sensors to determine the intended direction of travel. Turn buttons are located on the end of each handlebar. Pressing these buttons causes the front wheels to turn parallel to each other in the same direction and thus allows the walker to rotate in a circle about its rear wheels. Brake levers are also positioned on the handle grips.

The Walkmate [13] was developed to have two force sensing resisters embedded into the handlebar. These sensors are fixed with the handgrip directly so it can detect both pull and push pressure of the handlebar. Moreover, the force sensors embedded in handlebars offer a nature negative feedback loop of the motion control. On the one hand when the user wants to keep a constant velocity, the handle will be pulled to decelerate the walker if the walker is faster than the user. On the other hand, if the walker is slower than the user, the handlebar will be pushed to accelerate the walker.

Another walker that was developed by the Bioengineering Group at CSIC is the SIMBIOSIS [6]. This walker presents a multisensory biomechanical platform for predictive humanmachine cooperation. One of the sensory subsystems is the force interaction that is compound by a set of force sensors 
installed on the walker's forearm-supporting platform. The forces that are applied by the user on each forearm-support while he his walking are measured and the guidance information can be inferred. This turn out to be a natural and transparent interface that did not need previous training by the user. Other works are studying interfaces with force sensors are UTS [14], Chuy et al. [15], MARC [16].

Another kind of direct interfaces are the voice communications. To help the visually impaired, for example, it was developed by Gharieb at King Saud University [17] a walker that as the ability to steer following the user's voice and to avoid obstacles. The walker receives the voice command from the user and determines the required tasks. If the speed of the walker exceeds a maximum limit value an audio alarm will be activated, and when there is an obstacle it warns the user with an audio alarm, too.

On the other hand, there are the indirect interfaces that recognize user's movement and/or intent without requiring manual operations. Examples include visual recognition using cameras [18], and detection of human gait using pressure sensors [19].

A recent development, the JAIST active robotic walker (JARoW) by Lee et al [18], is a compact prototype that doesn't require artificial controls, because it has a pair of rotating infrared sensors detecting the location of the user's lower limbs. With a control algorithm developed by Lee et al the motion of walker is adjusted according to the user's walking speed and direction relative to the base frame of the walker, without employing electronic devices worn on the user body or manual controls such as joysticks or touchpad's.

As it was seen the major study of direct interfaces has been focused on force sensors, because unlike the joysticks, buttons and switches, for example, the torque sensors are natural and intuitive. When the joystick is moved in any direction during the gait, due to the foot strike or uneven terrain, vibrations may appear. Relatively to the buttons, switches and touch screens, they might be simpler and easy to control, but they have an intermittent or discrete operation that is mentally heavy for the user. Moreover, these interfaces may cause confusion for elderly users, which might result in an accident. The voice communication, for example, has an advantage of transferring effective high-level commands as a bilateral communication tool. However, there are critical problems such as interference and recognition to be resolved.

In the case of indirect interfaces, despite of the investigation that is being done recently, we need to incorporate an elaborate recognition algorithm and high performance devices into the visual recognition system. Human gait detection also requires an additional device worn on the human body and its outdoors environments remain a difficult problem. Despite these advances there are still unresolved questions in determining natural and efficient interfaces. Further, due to the limitations on current recognition and control algorithms and costs that need to be lower, there is room for further improvements and this has been a turning point for research. Additionally, it is very important to remember that these interfaces should not increase the cognitive burden or cause confusion and frustration for the elderly or lower limb disable users.

\section{THE NEOASAS PURPOSE}

The NeoASAS Project emerged in order to give continuity to the ASAS and SIMBIOSIS projects developed by the Bioengineering Group of CSIC, but with a commercial focus.

It is intended that the NeoASAS walker (Fig.1) has the same reliability and stability that the SIMBIOSIS walker [6], using a sensor system for the characterization of the humanrobot interaction during assistive gait. Nevertheless, in order to make it more robust and economic it requires a simplification of its sensorial configuration. Thus, it should be implemented with simple and low cost sensors and create a more aesthetic design of the walker. The interface of this new prototype must remain user-friendly without requiring additional cognitive capacities, since many users/patients have many needs related to these factors.

The NeoASAS walker, in its final state, will be an autonomous system capable of controlling the movement of the walker through the extraction of the users' movement intentions obtained by the integrated sensory system. This project is unique in the sense that it tries to improve the safety and stability of walkers, and avoid to inadequately resort to alternative devices such as wheelchairs, that have disabling effects, thus contributing and reinforcing to the maintenance or to the improvement of the physical and cognitive capabilities of the user, through functional compensation.

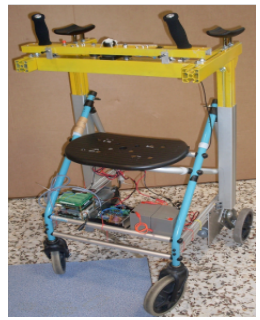

Fig. 1 The NeoASAS Smart walker.

In order to provide for walking support, the NeoASAS has to capture the direction that the user intends to go based on the user manipulation. The directional intent will be identified from physical manipulation because the user's directional intent and his physical manipulation usually are mutually consistent. In this paper, a novel interface is proposed to recognize the user's directional intention according to the manipulation of handles on the upper base support.

The development of this interface took into account that walking is a complex combination of omnidirectional motions, including forward and translational (right and left). The interface must have the capability to "read" and interpret all these kind of intended motions, to follow the user's movement, and to provide for a good walking support. Additionally, the novel interface has the advantage of using the forearm supports, improving safety during walking and helping to unload the lower limbs. Moreover, the user does not have to intentionally manipulate the device in order to set direction 
instructions and it is low-cost, if compared to other commonly used technologies in this field, such as force sensors or Brain Computer Interfaces.

The system proposed for evaluating the intentions of the user's walking movement is based on a joystick that is integrated into the upper base support at its middle. This joystick is the key of the interface. The device is based on an adapted conventional four-wheeled walker. The walker integrates a forearm support and the electronics heaviest components were mounted in the lower base of the walker. This walker allows the load of the lower limb weight on the walker and the decrease of the occurrence of uncontrolled movements of the joints of the arms. It was also noticed that the use of a walker with a smart control system allows the improvement of the gait patterns of disable users, and a greater stability in terms of the user's posture.

\section{A. Specifications of the human-machine interface}

During the SIMBIOSIS project it were observed and studied which efforts were the most important to drive the walker. According to these studies, in the NEOASAS project was proposed to use a new interface that consists on placing, at the centre of the upper base support, a joystick associated to two springs that is moved according to user's manipulationTo extract the signals from the joystick and then study them it was performed a series of tests with healthy volunteers, with no history of any dysfunction on either upper or lower limbs. These volunteers had to perform simple tasks like moving forward and then turn left or right. It is noteworthy that these tests were performed without any motorized system. This enables to evaluate the real interaction between the user and the upper base support of the walker without the interference of any control strategy. These signals enable to characterize the interaction between both agents.

When the user forces the handles, this slight movement is transmitted to the upper base support, mechanically coupled to the joystick that reads the user intention. When the user begins his gait, he has to slightly move the handlebar through the handles, moving the joystick, informing the walker which direction and velocity he wants to take. Hence, the user's efforts are successfully converted into small movements through this new interface, which is simpler, but is developed to be optimized for commercial implementation. In Fig. 2, it is depicted an image of the proposed interface.

The handlebar is a base and in its centre is integrated the joystick associated to springs, and in each one of the two extremes was placed a handle, so that the user can hold on to it (Fig. 2). The joystick is a robust and low cost device that does not require excessive use of electronics, and reduces the risk of failure. Besides being based on a joystick, this proposed interface is different from the one presented by Hashimoto [10] because the guidance of the walker is performed with two hands, and there is a support for the forearms, turning this purpose safer and more stable.

The joystick outputs three different signals (X,Y,Z), measured in Volts that specify the imposed movement described on the XYZ-axis attached to the joystick. However, in this work, only $\mathrm{Y}$ and $\mathrm{Z}$-axis are used.

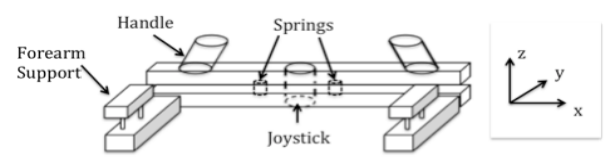

Fig. 2 Schematic of the NeoASAS interface with the joystick.

These signals are measured in Volts and are proportional to the rotation angle $\left(\theta_{\mathrm{i}}, \mathrm{i}=\mathrm{y}, \mathrm{z}\right)$ of the joystick around the $\mathrm{Y}$ and $\mathrm{Z}$ axes of the joystick that results from the force that is applied by the user on the handles (1) Signal Y is the angular rotation around the Y-axis and represents the straight or forward movement intention of the user. Signal $\mathrm{Z}$ is the angular rotation around the $Z$-axis and represents the curvilinear movement intention of the user. These are obtained as shown in (1).

$$
\begin{aligned}
& Y=0.125 x \theta_{y}+2.5 \\
& Z=0.125 x \theta_{z}+2.5
\end{aligned}
$$

Where $\theta_{y}$ is the rotation angle around the Y-axis and $\theta_{z}$ is the rotation angle around the Z-axis applied to the joystick. Both can vary within the range $\left[-18^{\circ}, 18^{\circ}\right]$.

These $\mathrm{Y}$ and $\mathrm{Z}$ signals are then sent to an architecture based on Matlab Real-Time xPC Target Toolbox to be processed.

To extract the signals from the joystick and then study them it was performed a series of tests with healthy volunteers, with no history of any dysfunction on either upper or lower limbs. These volunteers had to perform simple tasks like moving forward and then turn left or right. It is noteworthy that these tests were performed without any motorized system. This enables to evaluate the real interaction between the user and the upper base support of the walker without the interference of any control strategy. These signals enable to characterize the interaction between both agents.

The acquisition of these signals from the joystick allowed the identification of two main components of the signal. One represents the highest frequency noise caused by the vibrations introduced by the ground and wheels irregularities of the structure, and this component should be eliminated. The other one is the voluntary component that contains the information of the walking movement intentions of the user to guide the walker. This is the one that really interests to guide the walker. The typical joystick data, $\mathrm{Y}$ and $\mathrm{Z}$ signals are presented in Fig. 3.

As it can be seen, both signals present a high frequency component that has to be eliminated to clear the signal so it can be easier to identify the other component that characterizes the user's guidance intentions. The Y signals are zero at the beginning when the user is stopped, but when the user begins to walk forward, the signal becomes negative. Analogously, the $\mathrm{Z}$ signals have an amplitude close to zero, but around $t=17 \mathrm{~s}$, the user turns aside and a high amplitude peak is observed (Depending on the side that the user turns, the amplitude of the $\mathrm{Z}$ signal can be positive (right) or negative (left)). And at the same time the $\mathrm{Y}$ signal also changes being more positive. This tells us that when the user wants to turn aside the forward command is decreased. With 
the acquired data, it was performed an offline study for the definition of the signal processing methodology.
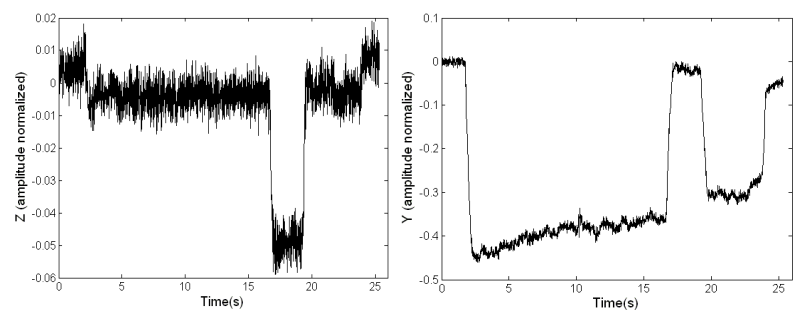

Fig. 3 Typical raw Joystick data ( $Z$ and $Y$ signals) obtained when the user is turning right.

\section{SignAl Processing METHOdOLOY}

This section presents the filtering strategy to attenuate/eliminate the higher-frequency component. After that, the joystick signal can be used for controlling the device's movement, converting user's intentions into motor commands. The data that was collected yields that the user's commands occur in a frequency range between 0 and $2 \mathrm{~Hz}$ in both $\mathrm{Z}$ and $\mathrm{Y}$ signals, and the rest of the higher-frequency components are related to noise caused by structure vibrations, etc (Fig.4). These last components are more accentuated in the $\mathrm{Z}$ signal.
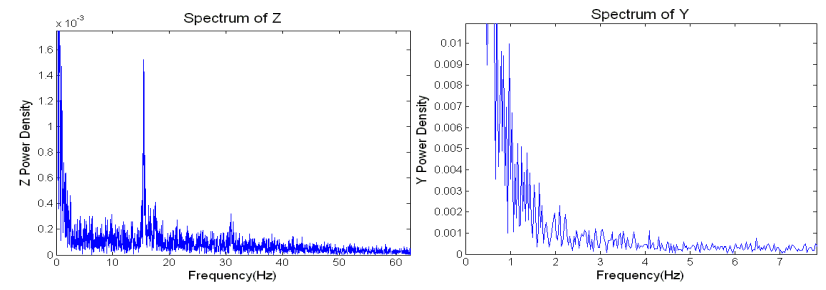

Fig. 4 Power Spectrum of the $\mathrm{Z}$ and $\mathrm{Y}$ signals.

According to this, the higher frequency components present in the signals can be eliminated with forth and back recursive digital, such as Butterworth filters, without causing phase distortion (Fig.5). This technique consists in filtering input data in both the forward and reverse directions; after filtering in the forward direction, the algorithm reverses the filtered sequence and runs it back through the filter, which yields precisely zero-phase distortion. However, this approach is not real-time implementable, but it will be the basis to evaluate the performance of the chose filter strategy.

The user's command will be modelled as a first order process (Taylor series), where the first derivative will be set as constant, considering the human movements are slow. The second derivative can be neglected since its maximum value is $4.16 \mathrm{E} 10^{-10} \mathrm{rad} / \mathrm{s}^{-3}$ for the $\mathrm{Y}$ signal and $2.48 \mathrm{E} 10^{-9}$ for the $\mathrm{Z}$ signal, and the sampling period $T s$ is $1 \mathrm{~ms}$. Thus, it was decided to use g-h filters. g-h filters are simple recursive tracking filters that estimate future position and velocity of a variable based on first order model of the process.

Measurements are used to correct these predictions, minimizing the estimation error [20]. The following equation set is the general form of a g-h filter [20]:

$$
\begin{gathered}
x_{k, k}=x_{k, k-1}+g_{k}\left(y_{k}-x_{k, k-1}\right) \\
\dot{x}_{k, k}=\dot{x}_{k, k-1}+\frac{h_{k}}{T_{s}}\left(y_{k}-x_{k, k-1}\right) \\
\hat{x}_{k+1, k}=x_{k, k}+T_{s} \cdot \dot{x}_{k, k} \\
\hat{x}_{k+1, k}=\dot{x}_{k, k}
\end{gathered}
$$

The filtering equations (2) and (3) estimate the current position, $x_{k, k}$, and velocity, $\dot{x}_{k, k}$, of the variable based on previous predicted position, $x_{k, k-1}$, and velocity, $\dot{x}_{k, k-1}$, taking the current measurement $y_{k}$ to account. Assurance on measures is weighted by gains $g_{k}$ and $h_{k}$. The prediction equations (4) and (5) predict the future position, $\hat{x}_{k+1, k}$, and velocity, $\hat{\dot{x}}_{k+1, k}$, based on a first order dynamic model of the variable. As g-h trackers consider a constant velocity model, predicted velocity is equal to the current one.

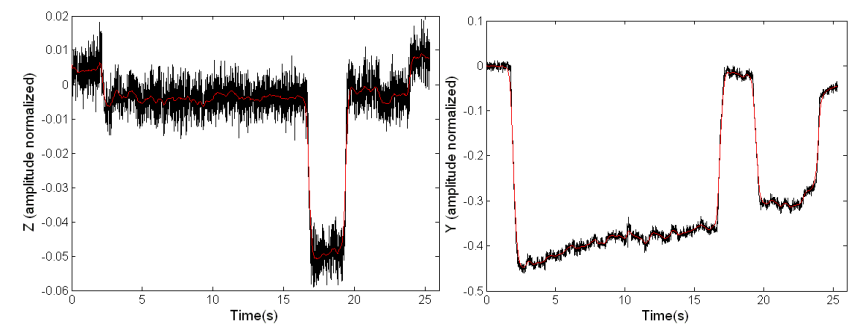

Fig. 5 In black it is represented the raw joystick data and in red it is represented the signal filtered with Butterworth filter.

g-h filters are affected by (a) systematic error, which is related to the constant velocity assumption, and (b) the measurement error, which is inherent to the joystick and measurement process. Typically, the smaller $g k$ and $h k$ are, the larger is the dynamic error and the smaller are the measurement errors [20]. Therefore, in designing a g-h filter there is a degree of freedom in the choice of the relative magnitude of the measurement and dynamic errors. To simplify the selection of the filter gains $(g k, k k)$ the BenedictBordner Filter (BBF) was applied. BBF minimizes the total transient error, defined as the weighted sum of the total transient error and the variance of prediction error due to measurement noise errors [21]. The BBf is the constant g-h filter that satisfies equation (6). As (6) related $g$ and $h$, the BBF has one degree of freedom. Because for $g$-h filters increasing the value of $g$ diminishes the transient error, so a larger $g$ makes the BBF to track higher frequencies.

$$
h=\frac{g^{2}}{2-g}
$$

\section{RESULtS}

For the selection of the $\mathrm{g}$ parameter, the Kinematic Estimation Error (KTE) was used (7). KTE evaluates the smoothness, response time, and execution time of a tracking algorithm [22].

$$
K T E=\sqrt{|\bar{\varepsilon}|^{2}+\sigma^{2}}
$$

$|\bar{\varepsilon}|$ and $\sigma^{2}$ are the mean and variance of the absolute estimation error between a desired signal and the measured 
signal [23]. Offline signal filtered using a Butterworth filter is used as the desired signal the estimators should track. The measured signal will be the signal filtered with the g-h filter.

To select the parameter $\mathrm{g}$, five individuals drove the walker without any motorization executing three experiments with five repetitions each. During the tests the signals of the joystick were acquired. These signals were then introduced off-line in the g-h algorithm using broad range of $g$ parameters. The result was processed by the KTE resulting on a cinematic error. The best solution, i.e. the one with the lowest KTE, was chosen for each user, experiment and repetition. With these results it was calculated the mean of the best solutions for the $\mathrm{g}$ parameter, as well as the mean of the delay between the input and the output of each case. Table I presents the mean values of the best solutions of $g$ parameter, delay between the original signal $(\mathrm{Y}$ and $\mathrm{Z})$ and the filter one with the $\mathrm{g}$-h and KTE for each joystick signal (Y and Z).

TABLE I

FILTER COEFFICIENT BASED ON THE KTE FOR Y AND Z SIGNALS. TABLE PROVIDES MEAN \pm STANDARD DEVIATION.

\begin{tabular}{|c|c|c|c|}
\hline Signal & $\mathrm{g}$ & Delay $(\mathrm{ms})$ & KTE $(\mathrm{rad} / \mathrm{s})$ \\
\hline$Y$ & $(46.64 \pm 21.90) \cdot 10^{-3}$ & 3 & $(6.14 \pm 2.28) \cdot 10^{-3}$ \\
\hline$Z$ & $(16.87 \pm 4.94) .10^{-3}$ & 20 & $(1.64 \pm 0.59) \cdot 10^{-3}$ \\
\hline
\end{tabular}

As it can be seen at Table I, $g$ of the $\mathrm{Z}$ signal compared with the $g$ parameter of the $\mathrm{Y}$ signal shows a lower value because the amplitude of its noise power density is much larger, requiring to be further filtered. In both cases, KTE and delay show very small values. The signals filtered (Fig. 6) shows to be of high quality for a human-machine interaction because its delay is 10 times inferior to human perception (200 ms), not causing prejudice to the human-machine interaction.

\section{CONCLUSION}

In this work it was presented a new method of user-walker interaction to extract the users' movement intentions. The interface is based on a joystick and is intended to be userfriendly, simple, efficient, low cost and with little electronics. A series of experiments were performed which showed the sensibility of the joystick to extract navigation commands from the user. These signals presented a higher frequency component that was attenuated by a Benedict-Bordner $\mathrm{g}$-h filter, where $\mathrm{g}$ is equal to $46.64 \mathrm{E} 10^{-3}$ for the $\mathrm{Y}$ and $16.87 \mathrm{E} 10^{-3}$ for the Z. This filter strategy is currently working in real-time and is programmed into the firmware installed on the NeoASAS walker. Future work includes the use of the joystick data processed with the filter presented in this work, as input signals for a control architecture that classifies, in real-time the navigational commands applied by the user during gait. An overall schematic is depicted in Fig. 7.

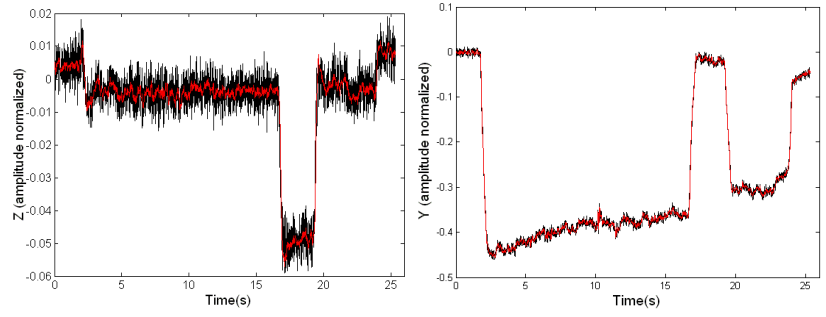

Fig. 6 In black it is represented the raw joystick data and in red it is represented the signal filtered with the $\mathrm{g}$-h filter (Benedict-Bordner).

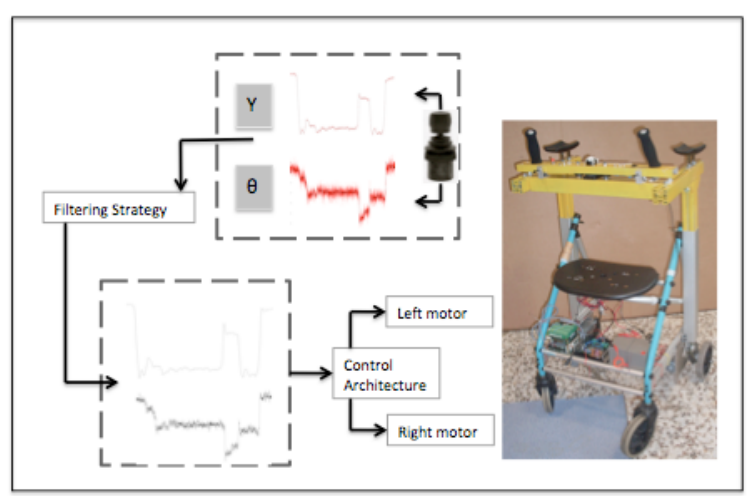

Fig. 7 Complete system architecture implemented on NeoAsas walker.

\section{REFERENCES}

[1] M. Hirvensalo, T. Rantanen, and E. Heikkinen, "Mobility difficulties and physical activity as predictors of mortality and loss of independence in the community-living older population," Journal of the American Geriatric Society, vol. 48, pp. 493-498, 2000.

[2] G. Lacey and K. Dawson-Howe, "Evaluation of Robot Mobility Aid for the Elderly Blind," in Proceedings of the Fifth International Symposium on Intelligent Robotic Systems, 1997.

[3] A. C. Morris, R. R. Donamukkala, A. Kapuria, A. Steinfeld, J. Matthews, J. Dunbar-Jacobs, and S. Thrun, "A robotic walker that provides guidance," in Proceedings of the 2003 IEEE Conference on Robotics and Automation, May 2003, pp. 25-30.

[4] Frizera A, Abellanas A, Ceres R, Pons JL, Raya R: Study and Characterization of Feet Kinematics in Walker Assisted Gait. Revista Iberoamericana de Automatica e Informatica Industrial (RIAI) 2009, 6(4): 74-84.

[5] Renpeng Tan, Shuoyu Wang, Yinlai Jiang, Kenji Ishida, and Masanori Nagano. Adaptive Controller for Motion Control of an Omni-directional Walker. Proceedings of the 2010 IEEE International Conference on Mechatronics and Automation August 4-7, 2010, Xi'an, China, pp. 156161.

[6] Frizera Neto A, Gallego JA, Rocon E, Pons JL, Ceres R. Extraction of user's navigation commands from upper body force interaction in walker assisted gait. BioMedical Engineering Online. 2010; 9 (37):1-16.

[7] M.J. McDonald. Active Research Topics in Human Machine Interfaces (Technical Report). Sandia National Labs., Albuquerque, NM (US), 2000.

[8] L. Bueno, F. Brunetti, A. Frizera, J.L. Pons. Human-Robot Cognitive Interaction. In: Wearable Robots: Biomechatronic Exoskeletons. 1 ed. John Wiley \& Sons, Inc., 2008, vol. 1, pp. 87-126.

[9] Ceres, R., Pons, J.L., Calderón, L., Mesonero-Romanos, D., Jiménez, V. F., Sánchez, X., Abizanda, P., Saro, B., and Bonivardo, G.. (2005) Andador activo para la rehabilitación y el mantenimiento de la movilidad natural. IMSERSO, Estudios I+D+I, 25:1 - 8 .

[10] H.Hashimoto, A.Sasaki, Y.Ohyama and C.Ishii. Walker with hand haptic interface for spatial recognition. In Proceeding of the ninth IEEE 
International Workshop on Advanced Motion Control, May 2006, pp. 311-316.

[11] G. Lacey, S. M. Namara, and K. M. Dawson-Howe., "Personal adaptive mobility aid for the infirm and elderly blind," Lecture Notes in Computer Science, 1458:211 - 220, 1998.

[12] A. J. Rentschler, R. Simpson, R. A. Cooper, M. L. Boninger. Clinical evaluation of Guido robotic walker. Journal of Rehabilitation Research Development, vol. 45, n. 9, 2008, pp. 1281-1294.

[13] F. Shi, Q. Cao, C. Leng and H. Tan. Based On Force Sensing-Controlled Human-Machine Interaction System For Walking Assistant Robot. Proceedings of the 8 th World Congress on Intelligent Control and Automation July 6-9 2010, Jinan, China, pp.6528-6533.

[14] S. McLachlan, J. Arblaster, D.K. Liu, J. V. Miro, L. Chenoweth. A Multi-Stage Shared Control Method for an Intelligent Mobility Assistant. Proceedings of the 2005 IEEE 9th International Conference on Rehabilitation Robotics June 28 - July 1, 2005, Chicago, IL, USA, pp. 426-429.

[15] O. Chuy, Y. Hirata, and K. Kosuge. Active Type Robotic Mobility Aid Control Based on Passive Behavior. Proceedings of the 2007 IEEE/RSJ International Conference on Intelligent Robots and Systems San Diego, CA, USA, Oct 29 - Nov 2, 2007, pp. 165-170.
[16] G. Wasson, J. Gunderson, and S. Graves, "Effective shared control in cooperative mobility aids," in Proceedings of the Fourteenth International Florida Artificial Intelligence Research Society Conference, pp. 1-5.

[17] W. Gharieb. Intelligent Robotic Walker Design. International Conference on Automation, Robotics and Autonomous Systems, June 2006, Sharm El Sheikh, Egypt.

[18] G. Lee, T. Ohnuma, N. Y. Chong. Design and control of JAIST active robotic walker. Intel Serv Robotics, 2010, pp. 125-135.

[19] M. Hirasawa, H. Okada, M. Shimojo. The development of the plantar pressure sensor shoes for gait analysis. Journal Robot Mechatron 20(3), pp. 324-330, 2007.

[20] E. Brookner. Tracking and Kalman Filtering Made Easy John Wiley and Sons, Inc 1998.

[21] Benedict, T.R.; Bordner, G.W. Synthesis of an optimal track-while-scan smoothing equations. IRE Trans. Automat. Control 1962, 7, 27-32

[22] E. Rocon, A. Ruiz, J. Moreno, J. Pons, J. Miranda, A. Barrientos. Tremor characterization. Algorithms for the study of tremor time series. International Conference on Bio-inspired Systems and Signal Processing - BIOSTEC Conference -Biosignals 2008, 355-360.

[23] Oppenheim AV, Schafer R: Discrete-Time Signal Processing PrenticeHall 1989 